\title{
Effects of Oil Heated with Gluten on Weight-Loss Dieting. I.
}

\author{
Nagao Totani ${ }^{1 *}$, Munkhjargal Burenjargal ${ }^{2}$ and Miho Yawata ${ }^{1}$ \\ ${ }^{1}$ Faculty of Nutrition, Kobe-Gakuin University (518 Arise, Ikawadani-cho, Nishi-ku, Kobe 651-2180, JAPAN) \\ ${ }^{2}$ Faculty of Chemistry, National University of Mongolia (P.O. Box 46A-442, Ikh Surguuliin Gudamj-1, Sukhbaatar District, Ulaanbaatar- \\ 210646, MONGOLIA)
}

\begin{abstract}
Fresh oil was heated for $20 \mathrm{~h}$ at $180^{\circ} \mathrm{C}$ with amino acids or gluten. A powdered diet (AIN93G; no fat) was mixed with $7 \mathrm{wt} \%$ of fresh oil (control), or supernatants of the heated oils described above, and fed to male Wistar rats for 12 weeks. No gross symptoms attributable to the heated oils were observed, but the gluten group showed a slow body weight increase; a significant difference was found in the weight after age 21 weeks in spite of diet consumption comparable to that of the control group. The serum of the heated oil groups showed a tendency toward lower values on various hematological measures, especially triacylglycerol and free fatty acid and toward higher values on aspartate aminotransferase (AST) and alanine aminotransferese (ALT), than those of the control group. All the rats except one in the gluten group had the same level of AST as those of the control rats, while the amino acid group included four rats with AST over $100 \mathrm{IU} / \mathrm{L}$, the highest value in the control group. The number of dark red patches found on the surface of the liver and histological evaluation also showed frequent damage to the livers of the amino acid group. The difference in toxicity between the two heated oils seems to be related to the molecular sizes of amino acids and gluten. Gluten or melanoidin produced during heating probably decreased or counteracted the cytotoxicity of thermally oxidized oil. It is expected that oil heated with gluten can be used as a safe and effective oil for humans on weight-reduction diets.
\end{abstract}

Key words: diet, body weight, gluten, triglyceride oligomer, melanoidin

\section{INTRODUCTION}

In recent years, the number of obese Japanese people older than 20 years has been consistently increasing ${ }^{1)}$. Body mass index values of 25 or greater was present in $19.8 \%$ of Japanese people in their twenties and $26.0 \%$ of those over age 70 , with the highest prevalence, $34.1 \%$, for those in their forties in 2005. It has also been reported that the number of Japanese people with metabolic syndrome is about 9.2 million among those 40 to 74 years old, with a potential for reaching 9.8 million, although obesity in women is relatively uncommon ${ }^{1)}$. Similar prevalences of metabolic syndrome are found in many countries, leading to an interest in the development of edible oils useful in weight reduction and the marketing of such oils by domestic and overseas companies. The mechanisms by which such oils may assist in weight reduction ${ }^{2)}$ are based on the following concepts: after absorption of oil through the microvilli of the small intestine, the absorbed fatty acids are not efficiently utilized; the content of fatty acids in glycerides is less than that of ordinary fats; or the fatty acids are easy to metabolize. Our approach to creating a safe and effective oil for weight-loss dieting is different from any of the above; we are investigating oils in which a part of the oil is not fully hydrolyzed by lipase, resulting in decreased absorption and increased amounts of excreted oil.

The ideal oil for weight-loss dieting should be a safe and inexpensive substance, should contain appropriate amounts of the fatty acids needed in human nutrition, should help to decrease the weight of an obese subject to the standard weight during normal intake of the oil, and should not differ from ordinary oil in palatability, smoothness in the mouth, sensations during digestion, or excretion. Too-rapid decrease of body weight through dieting is

*Correspondence to: Nagao Totani, Department of Nutritional Physiology, Faculty of Nutrition, Kobe-Gakuin University, 518 Arise, Ikawadani-cho, Nishi-ku, Kobe 651-2180, JAPAN

E-mail: totani@nutr.kobegakuin.ac.jp

Accepted March 12, 2008 (received for review January 28, 2008)

Journal of Oleo Science ISSN 1345-8957 print / ISSN 1347-3352 online

http://www.jstage.jst.go.jp/browse/jos/ 
dangerous and is often connected with body weight rebound. However, too slow a decrease in body weight is also undesirable. In addition, for a special oil for weightloss dieting to be acceptable to consumers, its physical properties during cooking, oxidation and storage stabilities, and handling of the oil should be equivalent to or better than those of ordinary oil.

Billek $^{3}$ et al. separated by column chromatography sunflower oil used in the industrial production of fish fingers into polar and nonpolar fractions. Then rats were fed a diet containing $20 \%$ of each fraction for 18 months. The nonpolar fraction caused a highly significant reduction in weight gain of the animals as compared with fresh sunflower oil but had only an insignificant detrimental effect upon the many biochemical, histological, and clinical parameters assessed.

We have focused on lipase-resistant triglyceride oligomers as effective compounds for weight-loss dieting. As a condition for the production of an oil for dieters, fractionation of heated oil by column chromatography or other methods is not desirable because large amounts of elution solvents and time are needed, resulting in high cost of the product. On the other hand, heating of oils presents problems in terms of generation of toxic compounds. In the present study, oil was heated with an amino acid mixture or gluten to evaluate the inhibition of toxicity by these substances and/or melanoidin formed from them ${ }^{4}$.

\section{EXPERIMENTAL}

\subsection{Materials}

2.1.1 Oil

The fresh oil, commercial frying oil, was made of fresh soybean and rapeseed oils. One liter of this oil was heated at $180^{\circ} \mathrm{C}$ for $20 \mathrm{~h}$ in a 2-L four-necked flask under stirring with Gln, Gly, Ala, Tyr, Arg, Pro, Thr, Asp (Nacalai Tesque, Inc., Kyoto), 200 ppm each, and $1 \mathrm{wt} \%$ gluten from wheat (Nacalai Tesque, Inc., Kyoto), respectively. Experimental oils, thus obtained, were allowed to stand at a room temperature for a day to precipitate solid materials, and each supernatant was employed for an animal experiment.

2.1.2 Diets

A commercial pelleted diet (Labo MR Stock, Nihon Nosan Kohgyo, Japan) and a powdered AIN93G diet without fat (Japan Clea, Tokyo) were purchased. Using a blender, the latter was mixed uniformly with $7 \mathrm{wt} \%$ of experimental oils and fresh oil, respectively. The three kinds of diets, thus prepared, were handled and provided as described in our paper ${ }^{5-7)}$. All the diets were used after radio-sterilization.

\subsubsection{Chemical Analyses}

Methods for chemical analyses of oil were the same as in our previous papers ${ }^{8,9}$. The fatty acid compositions of the fresh oil analyzed as previously ${ }^{8,9)}$ were as follows: myristic acid $0.1 \%$; palmitic acid $8.4 \%$; palmitoleic acid $0.1 \%$; stearic acid 3.3\%; oleic acid 38.2\%; linoleic acid 39.2\%; and $\alpha$ linolenic acid $6.5 \%$; eicosenoic acid $0.6 \%$; others $3.6 \%$.

\subsection{Animals}

Male Wistar rats aged 9 weeks were obtained from Japan SLC, Inc., Shizuoka, Japan, and were housed separately in wire cages at $24 \pm 2{ }^{\circ} \mathrm{C}$ and humidity $50 \pm 10 \%$, with light from 7:00 to 19:00 at Japan SLC, Inc., Animal Experiment Center, Shizuoka, Japan. Animal care and handling were in accordance with the Ethical Agreement Concerning Care and Use of Laboratory Animals for Research and Education, Kobe-Gakuin University.

\subsection{Procedure of animal experiment}

Twenty-four animals were maintained on Labo MR Stock for 1 week of adaptation; animals were then divided into three groups (8 rats/group) by Statlight System, Yukms, Tokyo, Japan in order to make average body weights similar and minimize standard deviation in body weight. Two groups were fed a diet containing $7 \mathrm{wt} \%$ oil heated with amino acids or gluten, and the third group was fed a diet containing $7 \mathrm{wt} \%$ fresh oil. All animals were allowed feed and water ad libitum throughout the experiment. Autoxidation of oil in the diet was avoided by supplying a fresh diet daily. Body weight and the amount of feed ingested were determined weekly. After 12 weeks, a fasting period of $18 \mathrm{~h}$ was imposed prior to the administration of anesthesia. Serum was obtained from blood drawn from the abdominal aorta. Livers, kidneys, and retroperitoneal tissue were excised, weighed, and examined.

\subsection{Hematological analyses}

All the determinations were made as in our previous papers ${ }^{5,6)}$.

\subsection{Histological evaluations}

All the methods were the same as those in our previous papers ${ }^{5,6)}$.

\subsection{Statistical analysis}

All the values obtained from animals are revealed as mean \pm SD. Data from 8 animals each for experimental and control groups were analyzed using Student's t-test for unpaired observations and results were considered significant at $p<0.05$.

\section{RESULTS}

\subsection{Oil analyses}

Chemical properties of the oils are shown in Fig. 1. After heating, peroxide value (POV), carbonyl value (COV), and polar compound content $(\mathrm{PC})$ increased and triacyglycerol 


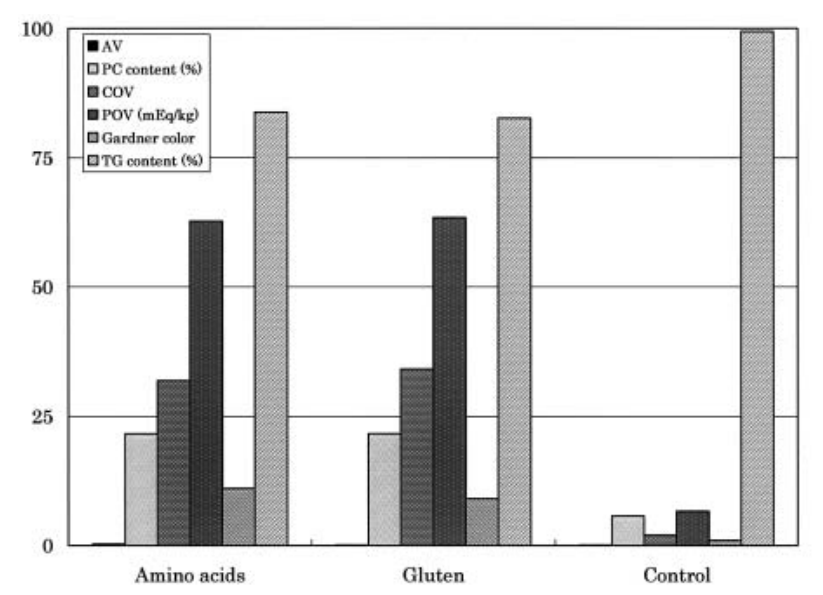

Fig. 1 Chemical Properties of Oils Heated with Amino Acids or Gluten.

content (TG) decreased. Acid value (AV) stayed unchanged because the oil did not contain water. Both experimental oils changed color due to the amino carbonyl reaction; the oil heated with amino acids browned drastically in spite of the small amount of amino acids added. The oil heated with gluten had a mild odor with a very slight paint-like odor.

\subsection{Growth on sample oils}

All the rats appeared to grow normally: no diarrhea, seborrhea, dermatitis, or excessive hair loss was observed after the administration of any diet. Body weight also increased normally (Fig. 2), but the difference between the three groups gradually increased after age 17 weeks, and the weight of the gluten group was significantly lower than that of the control group at ages 21 and 22 weeks. Because

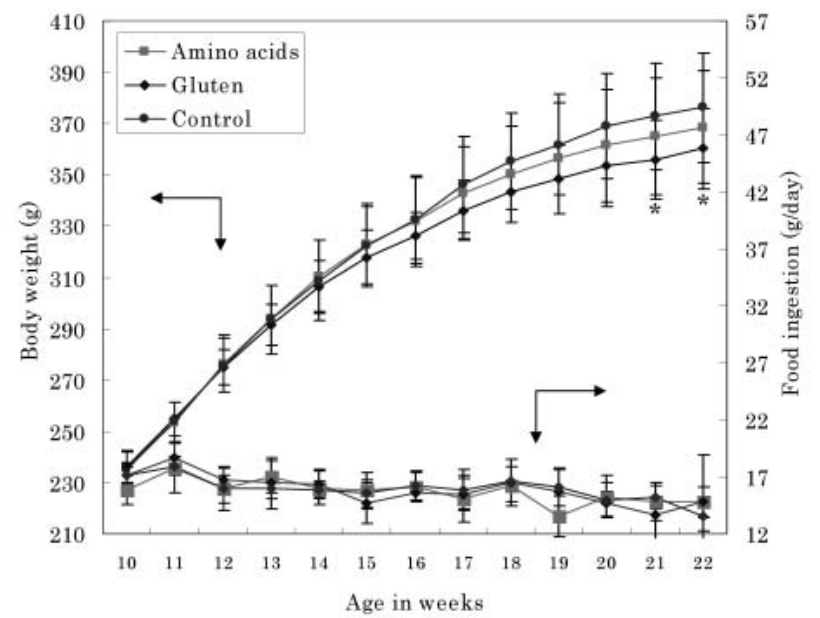

Fig. 2 Growth of Wistar Rats Fed a Diet Containing Oil Heated with Amino Acids or Gluten. ${ }^{*} p<0.05$, significantly different from the value of control (unpaired t-test).
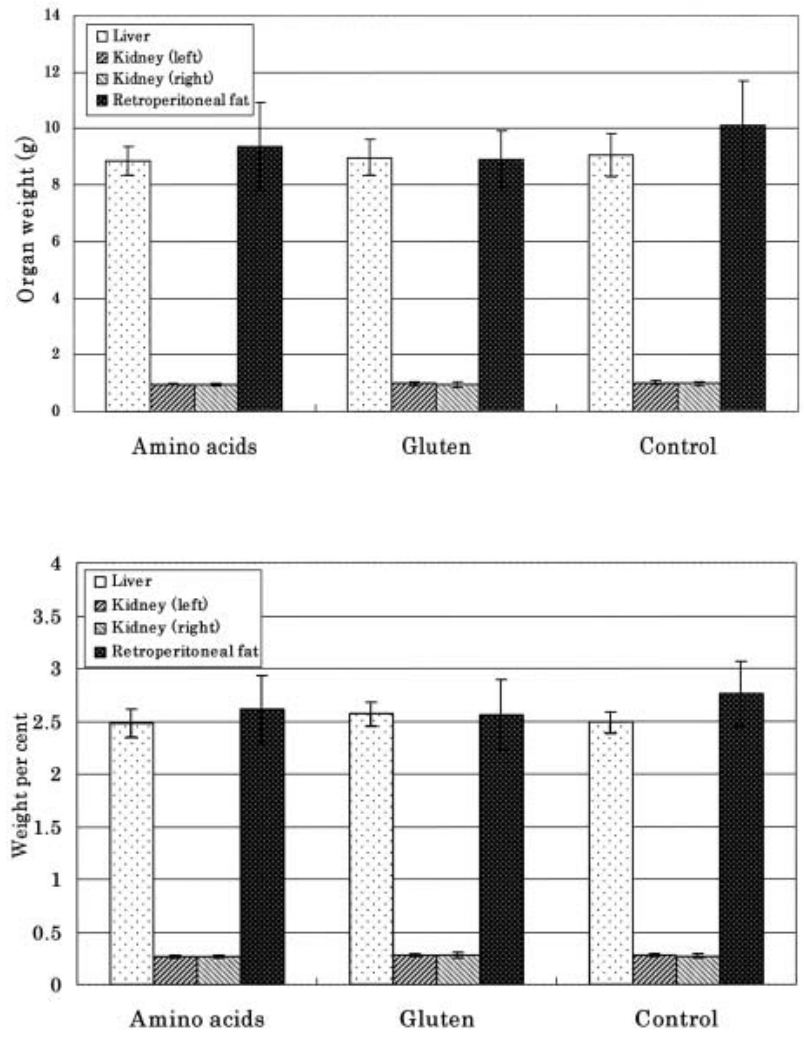

Fig. 3 Weights and Weight Percentages of Organs from Wistar Rats Fed for 12 Weeks a Diet Containing Oil Heated with Amino acids or Gluten.

of age, the amount of food ingestion decreased slowly, without any statistically significant difference among the three groups. This implies that the experimental oils did not have deleterious effects on the appetite of the rats.

The weights of organs excised are shown in Fig. 3. No differences in the weights of the liver, kidneys, and retroperitoneal tissues, and those weight percentages vs. body weights between groups were detected. However, in the experimental groups, there was a decreasing tendency in the weight of retroperitoneal fat, which is closely related to body weight.

\subsection{Hematological analyses}

As shown in Fig. 4, there was no difference between the levels of glucose, phospholipids, and cholesterol among the groups. However, triacylglycerol levels differed significantly between the amino acid group and the control group, and free fatty acid levels of the two heated oil groups were significantly different from those of the controls. The average magnitude of AST and ALT was in the order amino acid group, gluten group, and control group, although no significant difference was found among them. However, individual AST and ALT values corresponded well with the degeneration of the liver described below. 


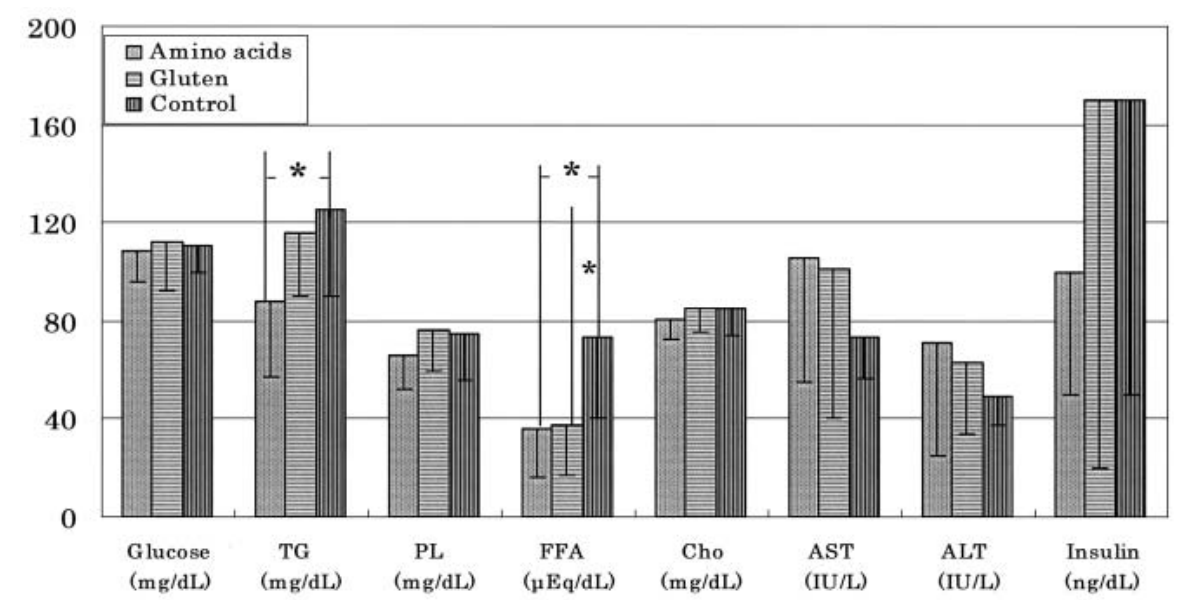

Fig. 4 Hematological Values in Wistar Rats Fed for 12 Weeks a Diet Containing Oil Heated with Amino acids or Gluten.

${ }^{*} p<0.05$, significantly different from the value of control (unpaired t-test).

Table 1 Organ Damages of Rats Fed a Diet Containing Oil Heated with Amino Acids or Gluten at $180^{\circ} \mathrm{C}$ for $20 \mathrm{~h}$.

\begin{tabular}{lccc}
\hline & Amino acids & Gluten & Control \\
\hline $\begin{array}{l}\text { Occurrence of AST higher than maximum } \\
\quad \text { AST of control/8 rats }\end{array}$ & $4 / 8$ & $1 / 8$ & - \\
Occurrence of ALT higher than maximum & $3 / 8$ & $2 / 8$ & - \\
$\quad$ ALT of control/8 rats & & & \\
$\quad$ No. of rats with dark-red patches on the & $6 / 8$ & $2 / 8$ & $1 / 8$ \\
$\quad$ liver/8 rats & & & \\
$\quad$ No. of rats with damage in the liver/8 rats & $7 / 8$ & $1 / 8$ & $1 / 8$ \\
No. of rats with damage in the kidneys/8 rats & $1 / 8$ & $1 / 8$ & $0 / 8$ \\
\hline
\end{tabular}

The occurrence of AST and ALT values that were higher than the maximum AST (100 IU/L) and ALT (68 IU/L) of the control group was determined for each group, as shown in Table 1.

\subsection{Visible changes in livers and kidneys}

Many dark red patches due to dotted bleeding were observed on the surfaces of the livers ${ }^{5)}$ from the amino acids group, suggesting degeneration of inner tissues (Table 1). A control rat and two rats in the gluten group also had patches, but their numbers were small. The AST level of individual rats also corresponded well with the number of dark red patches, so that the percentage of rats with dark red patches and that of rats having high AST showed similar tendencies.

No differences in color and size were observed in the kidneys of experimental vs. control rats, and no patches were observed in any group.

Microscopic evaluation showed frequent necrosis and bleeding of the liver in the amino acid group, but not in the gluten group and the controls. Compared to the liver, the kidneys did not show frequent or severe degeneration in any group (Table 1), but a rat in the gluten group also showed damage as in the amino acid group so that further experiments are needed to judge the safety of the oil heated with gluten.

\section{DISCUSSION}

Both heated oils were inferior in chemical properties to fresh oil (Fig. 1), but not to the degree where acute toxicity would be expected. The quality of the oil was similar to that found frequently in oil extracted from batter coatings of commercially deep-fried products ${ }^{10)}$. It is common understanding in Japan ${ }^{11-13)}$ that edible oil should have POV $<30$ $\mathrm{mEq} / \mathrm{kg}$ for the safety reason. It has also been reported ${ }^{14)}$ that properties such as POV, COV, PC, AV, and TG have little to do with cytotoxicity causing degeneration in the liver and kidneys, but that low molecular-weight compounds 
were possibly related to cytotoxicity. Gabriel et al. ${ }^{15)}$ reported that overt symptoms of heated fat toxicity did not develop in the Wistar rats received thermally oxidized olive oil, but in the rats given the distillable fraction of the oil. As described in 3.1, the oil heated with gluten had a mild odor, and showed little cytotoxicity in the present study. It will be reported in our next paper what happens to the oil under heating with gluten.

The amounts of oil ingested by rats in all groups decreased gradually, with no significant difference between groups, and reflected normal appetite (Fig. 1). However, the body weight increases of the heated oil groups were smaller than that of the control group, and the weight of the gluten group was about $5 \%$ less than that of the controls after age 21 weeks (the $11^{\text {th }}$ experimental week), with a significant difference. The three groups did not show significant differences in the weights and percentages of liver, kidneys, and retroperitoneal fat (Fig. 3), but the heated oil groups had a tendency toward decreasing weight of retroperitoneal fat. In the present study, liver, kidneys, and retroperitoneal fat were observed. But other organs, such as lung and testicle, should have also been investigated to ensure the safety of the oil. When the safety of the oil heated with gluten is thoroughly confirmed, it seems to be a promising oil for weight-loss diets because the body weight increase was suppressed during the experimental period without deterioration in appetite.

Hematological analysis of the gluten group showed values not significantly different from those of the control group except for free fatty acid level (Fig. 4). The triacylglycerol level of the gluten group was not so decreased and was higher than that of amino acid group, although the gluten group weighed less than the control group. As the supernatant of the heated oil was employed in the animal experiment, effects of trace amounts of heated gluten and amino acids remaining in the oil should be taken into account. Because the average AST and ALT of the heated oil groups did not clearly reflect organ damage, high values from individual rats were taken to correlate with the occurrence of cytotoxicity (Table 1). The liver damage was obviously higher in the amino acid group than in the gluten group, although the latter group was not free from the cytotoxicity.

The decreased nutritional value of thermally oxidized oils has been extensively reported ${ }^{16-19)}$. Marquez-Ruiz et $a l .^{20)}$ investigated the contribution of hydrolysis and absorption to the reduced digestibility of heated-oxidized oils and reported difficulties in the hydrolysis of complex glyceridic molecules included in the oil. Also, their data suggested that the extent of hydrolysis undergone in vivo was closely dependent on the amount and degree of alteration of the dietary fat. In the case of non-polar dimer fatty acids $^{3)}$, the low digestibility value may be associated in part with difficulties during the absorption process.
The major portion of the oil heated with gluten consisted of intact triglycerides at about $80 \%$. Thus, triglyceride oligomers that are not fully hydrolyzed by lipase amount to $20 \%$ at most. We are accustomed to intake of such oil through commercial products such as pork cutlet, in which breaded raw pork is deep-fried while exuding much juice containing protein and other components into the frying oil. It is reasonable to ask why, if people consume such oils, they nevertheless suffer from obesity. One of the reasons seems to be in the batter coatings. We will report results on this point in an upcoming paper. As current eating habits involve the consumption of oil similar to oil heated with gluten, it is hard to find problems with palatability, feeling, and repletion after ingestion, or with excretion. It is not clear yet by what mechanism gluten inhibited the cytotoxicity of heated oil. We suggest that the formation of low-molecular-weight compounds causing cytotoxicity, generated through decomposition of thermally oxidized oil, was inhibited and/or decreased by gluten ${ }^{14}$. The difference in the effect of oils heated with amino acids and gluten on cytotoxicity was conjectured to be due to the molecular size of the melanoidin formed from them ${ }^{4)}$.

The antioxidant property of melanoidin is known ${ }^{21}$. However, the POV and COV of oil heated with gluten were not especially low (Table 1), so this antioxidant property appeared not to occur under heating at $180^{\circ} \mathrm{C}$. On the other hand, high-molecular-weight melanoidins are absorbed to only a very limited extent $(<0.5 \%)^{4)}$, and thus they exert food fiber-like actions in the large intestine. In experiments in $\operatorname{rats}^{22,23)}$, these melanoidins improve intestinal flora; shorten the staying period of food in the intestine; increase the water content and weight of feces; and suppress changes in blood sugar level.

In conclusion, it was suggested that oil heated with gluten was feasible as a safe oil for weight-loss dieters that decreases body weight at an appropriate rate. It is also obvious, however, that the chemical properties of the oil must be improved to make them comparable to those of ordinary fresh oil in order for the product to be marketed successfully.

\section{ACKNOWLEDGMENT}

We are very grateful to Mr. Nobuzoh Iwa, Pathology Department, Municipal Hospital, Kashihara, Osaka, for the interpretation of histological specimens of liver and kidneys. This work was supported partly by a grant from the Life Science Center of Kobe-Gakuin University.

\section{References \\ 1. National Survey of Health and Nutrition, Japan}


(2007).

2. Jpn. Oil Chem. Soc. ed. Yushi, Shishitu no Kiso to Ohyou, Jpn. Oil Chem. Soc. Tokyo, pp.236-238 (2005).

3. Billek, G. Heated oils-chemistry and nutritional aspects. Nutr. Metab. 24, 200-210 (1979).

4. Takamiya, K. Iro kara mita Shokuhin no Science, Science Forum, Tokyo, pp. 183-191 (2005).

5. Totani, N.; Ojiri, Y. Mild ingestion of used frying oil damages hepatic and renal cells in Wistar rats. J. Oleo Sci. 56, 261-267 (2007).

6. Totani, N.; Ojiri, Y. Thermal deterioration of oil and frying foodstuffs. J. Oleo Sci. 56, 543-551 (2007).

7. Totani, N.; Yawata, M.; Ojiri, Y.; Fujioka, Y. Effects of trace acrylamide intake in Wistar rats. J. Oleo Sci. 56, 501-506 (2007).

8. Totani, N.; Kuzume, T.; Yamaguchi, A.; Takada, M.; Moriya, M. Amino acids brown oil during frying. $J$. Oleo Sci. 55, 441-447 (2006).

9. Totani, N.; Satoh, K.; Tsuji, S.; Yamaguchi, A. Effects of deteriorated frying oil in SHR/NDmc-cp rats. J. Oleo Sci. 55, 283-289 (2006).

10. Totani, N.; Ohno C.; Yamaguchi, A. Is the frying oil in deep-fried foods safe? J. Oleo Sci. 55, 449-456 (2006).

11. Itakura, S. Shishitu no kagaku, Asakura-shoten, Tokyo, pp. 164-167 (2001).

12. Ohyanagi, Y.; Inoue, M. Kassei-sanso to Roukagensho, Kyoritu-shuppan, Tokyo, pp.48-52 (2001).

13. Gotoh, N; Wada, S. The importance of peroxide value in assessing food quality and food safety. J. Am. Oil Chem. Soc. 83, 473-474 (2006).

14. Totani, N.; Munkhjargal, B.; Yawata, M.; Ojiri, Y. Chem- ical properties and cytotoxicity of thermally oxidized oil. J. Oleo Sci. 57, 153-160 (2008).

15. Gabriel, H.G.; Alexander, J.C.; Valli, V.E. Nutritional and metabolic studies of distillable fractions from fresh and thermally oxidized corn oil and olive oil. Lipids 13, 4955 (1978).

16. Artman, N.R. The chemical and biological properties of heated and oxidized fats. Adv. Lipid Res. 7, 245-330 (1969).

17. Perkins, E.G. Chemical, nutritional and metabolic studies of heated fats. Rev Fr. Corps Gras. 23, 257-262 (1976).

18. Billek, V.G. Die Veraenderungen von Nahrungsfetten bei hoeheren Temperaturen. Fat Sci. Technol. 94, 161172 (1992).

19. Combe, N.; Constantini, M.J.; Entressangles, B. Lymphatic absorption of nonvolatile oxidation products of heated oils in the rats. Lipids 16, 9-14 (1981).

20. Marquez-Ruiz, G.; Perez-Camino, M.C.; Dobarganes, M.C. Evaluation of hydrolysis and absorption of thermally oxidized olive oil in non-absorbed lipids in the rats. Ann. Nutr. Metab. 37, 121-128 (1993).

21. Chuen, N.V. Maillard reaction in food processing, process-induced chemical changes in foods, Prenum Press, p. 213 (1998).

22. Gomei, N. Shokuhin no Eiyou, Seiri-kinousei, Shokuhin no Hinshitu to Seibunkan-hannou, Kodansha, Tokyo, p. 237 (1990).

23. Kaminogawa, S.; Tanokura, M. Shokuhin no Kagaku, Tokyo Kagaku Dojin, Tokyo, p.106 (2005). 